\title{
Modeling of Spatial Configuration of the Loop and Calculation of the Length of the Thread in the Loop of the Tunting Binding
}

\author{
Umarova Mavludahon Nazirovna* \\ Candidate of Technical Sciences, Associate Professor. Department of "Materials Science and Technology of \\ New Materials”, Andijan Machine-Building Institute.Uzbekistan, Andijan city.

\begin{abstract}
*Corresponding Author:Umarova Mavludahon Nazirovna, Candidate of Technical Sciences, Associate Professor Department of "Materials Science and Technology of New Materials", Andijan MachineBuilding Institute.Uzbekistan, Andijan city.
\end{abstract}

\begin{abstract}
The problems of determining the length of the thread in the loop of a knitted fabric, worked out plush knitwear on the basis of smooth surface with a sequential alternation of natural silk thread and cotton yarn are considered. The length of the thread in the loop is considered in three-dimensional space, taking into account the geometric parameters of natural silk yarn, which allows us to predict the length of the thread in the loop with high accuracy both the idea of fabrics containing silk yarn and for knitted fabrics made from traditional textile yarns.
\end{abstract}

Keywords:: knitted fabric using natural silk thread, three-dimensional space, geometric loop model, spatial configuration of the loop, plush knitwear, length of the thread in the loop, weaving, surface density of the fabric.

\section{INTRODUCTION}

Methods for the manufacture of knitted fabrics from various types of raw materials are based on existing types of main weaving webs and their derivatives. The parameters of the structure of knitwear depend on the size, shape and relative position of the elements of the structure of knitwear loops, drafts, broaches relative to each other and the size of needle-platinum products, thereby determining the properties of the produced fabrics (length of thread in the loop (DNP), loop density, surface density of the web, dimensions, shrinkage, possible defects, etc.). In scientific research on determining the length of a thread in a loop, models of geometric similarity of a loop structure [1] or models of the shape of an elastic thread in a loop based on the theory of flexible elastic rods [2,3] are used. Existing models for determining DNP cannot accurately describe its length for threads made of natural silk yarn, and for the design of non-traditional types of knitted fabrics, a dependence is necessary that takes into account the spatial position and the curved thread in the loop.

This article proposes a calculation of the length of a thread in a loop of a knitted fabric, considered in a condition of equilibrium using a model of geometric similarity of a loop structure. A feature of determining the length of a thread in a loop, in comparison with other methods, is that the loop is considered in three-dimensional space taking into account its geometric parameters, and solving the problem reduces to calculating the integral in elementary functions.

\section{RESEARCH MethoD}

Since the raw material for the manufacture of yarn is natural silk yarn [4], the knitted fabric produced is plush weaving on the basis of a pattern with sequential alternation of rows of cotton yarn and natural silk yarn, a set of loops is carried out simultaneously with needles [5]. Modeling of the spatial configuration and calculation of the length of the thread in the loop involves the following system of assumptions:

- Calculation of the length of the thread in the knitwear loop is considered in a conditionally equilibrium state using a model of geometric similarity of the loop structure;

- The length of its center line is taken as the length of the thread;

International Journal of Research Studies in Electrical and Electronics Engineering (IJRSEEE)Page | 12 
- The average diameter $\mathrm{d}, \mathrm{mm}$, the threads in all sections of the loop are taken equald $=\frac{d_{p}+d_{y}}{2}$

- The diameter of the thread in the free state is equal to the thickness and width of the thread (crosssection is a square) and equals to the calculated diameter $\mathrm{dp}, \mathrm{mm}$. The diameter of the thread in a deformed state (applying a load along the axis of the thread) is equal to the nominal diameter dy, $\mathrm{mm}$.

Consider the projection of a loop of silk yarn of constant thickness and width $d$ on the XOY plane (Fig. 1 a. Top view) and on the XOZ plane (Fig. 1 b, side view). Line 0 , D is the axis of symmetry of the loop.

Half the length of the loop CD is symmetric with respect to its center; accordingly, it suffices to find the arc length of the circle of the speaker equal to the circle BD to calculate the length of half the length of the loop (Fig. 1). The roundness of the needle and platinum arches of the loop can be approximated by the arcs of circles $\mathrm{AC}$ and $\mathrm{BD}$, and the loop of sticks between the arcs - a segment of a straight line AB (Fig. 1a).

The axis OX is chosen parallel to the axis of symmetry of the loop and passes through point $\mathrm{C}$. The middle of the arc of the circle of the speaker is denoted by N. To determine the length of the half-loop of silk yarn having a width d, it is enough to find the length of its mid equidistant curve, respectively, the points $C, H, A, B$ belong to this equidistant (Fig. 1 a).

We consider only the equidistant curve CD (Fig. 2), where the center of the circle of the left arc of the loop coincides with the origin.

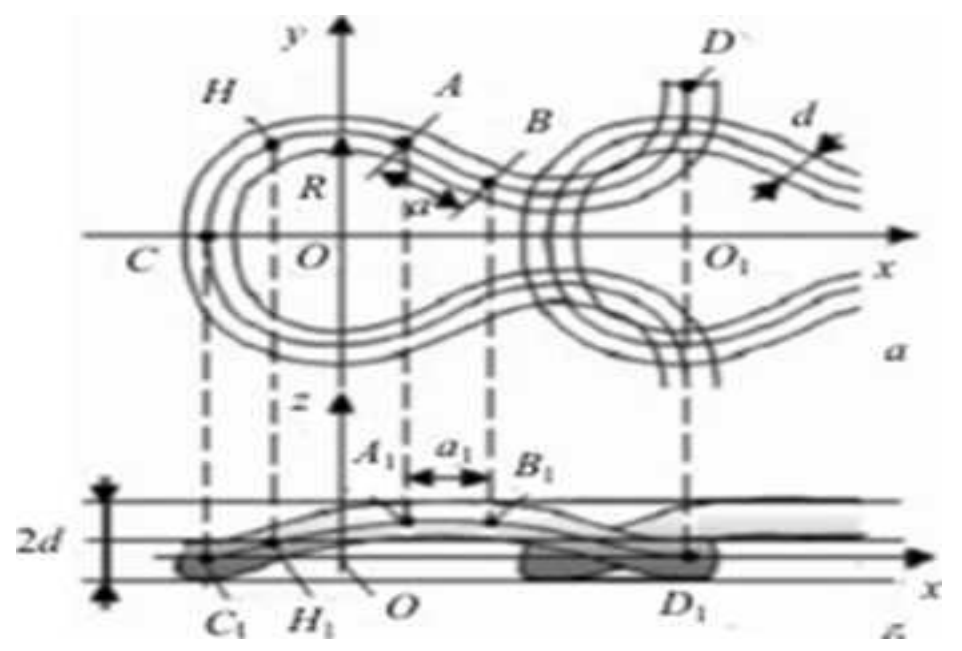

Figure1. The model of the shape of the thread in the loop: a-top view; $b$-view from the side.

Since at the points A and B the angular coefficient of the circles and the straight line coincides, to find it we denote the acute angle between the straight line $\mathrm{AB}$ and the OX axis by "-a", then the angle between the straight line OA and the OX axis is $\pi / 2-\alpha$.

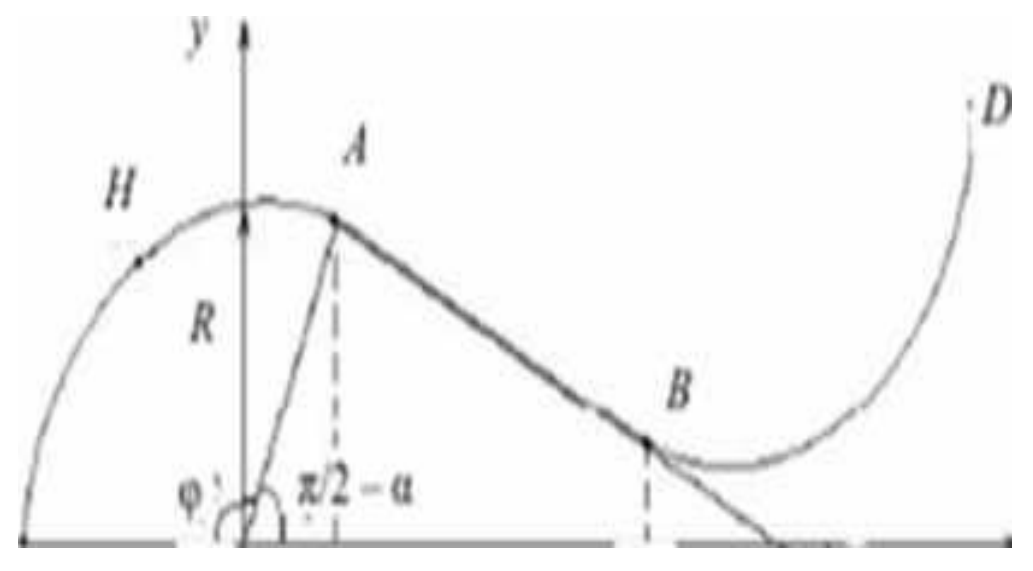

Figure2. Modeling the projection of a half loop. 
We use the coordinate system in parametric form. Then the part of the half-loop described by the arc of a circle has

$\left\{\begin{array}{c}x=R \cos \varphi ; \\ y=R \sin \varphi ;\end{array} \frac{\pi}{2}-\alpha \leq \varphi \leq \pi\right.$,

The where $\mathrm{R}$ is the radius of the arc of the circle obtained by approximating the loop of the thread.

Since the arc of a circle CA has the equation

$y=\sqrt{R^{2}-x^{2}}$,

then the ordinate of point $\mathrm{A}$ for $\boldsymbol{x}=R \sin \alpha$ is equal to, $\mathrm{y}=\mathrm{R} \cos \alpha$.

Let us describe the location of the arc SA in the space

$\left\{\begin{array}{l}x=R \cos \varphi ; \\ y=R \sin \varphi ; \quad \frac{\pi}{2}-\alpha \leq \varphi \leq \pi . \\ z=z(\varphi) ;\end{array}\right.$

From fig. $1 \mathrm{~b}$ it can be seen that the tangents to the curve CA located in the XOYZ space at points $\mathrm{C}$ and $A$ are horizontal, then at these points the derivatives of the function $z=z(\varphi)$ for the corresponding values of the parameter $\varphi$ will be equal to zero. Since the SN curve is smooth (a continuously differentiable curve) at the point $\mathrm{C}$, the value of the parameter $\varphi$ is $\pi$, then

$z=b(\varphi-\pi)^{2}$.

Moreover, in the coordinate system of the applicate at point $\mathrm{C}$ is zero. For a given choice of the curve, $Z^{\prime}(\varphi)=2 b(\varphi-\pi)=0$. Since the point $H$ - is the middle of the arc SN, in the coordinate system $\varphi$ $O Z$ the coordinates of the points $H$ and $C$ (Fig. $1 \mathrm{~b}$ ) have the forme in a parametric form form

$H\left[\varphi=\frac{3 \pi}{4}-\frac{\alpha}{2} ; z=\frac{d}{2}\right], C[\varphi=\pi ; z=o]$.

Substituting the coordinates of these points in equation (2), we obtain:

$\frac{d}{2}=b\left\{\frac{2 \pi}{4}-\frac{\alpha}{2}-\pi\right\}^{2}$.

From equation (3) we find the value of the coefficient

$b=\frac{d}{2\left\{\frac{\pi}{4}+\frac{\alpha}{2}\right\}^{2}}$.

In view of (1) and (2), the equation of the part of the $\mathrm{CH}$ loop has the form

$\left\{\begin{array}{c}x=R \cos \varphi ; \\ y=R \sin \varphi ; \\ z=b(\varphi-\pi)^{2} ;\end{array}\right.$

It is known that the length of the spatial curve is

$L_{C H}=\int_{\varphi 1}^{\varphi 2} \sqrt{\left[x^{\prime}(\varphi)\right]^{2}}+\left[y^{\prime}(\varphi)\right]^{2}+\left[z^{\prime}(\varphi)\right]^{2} d \varphi$.

We determine the length of the SN section by the formula (6) on the segment $\left[\frac{3 \pi}{4}-\frac{\alpha}{2} ; \pi\right]$.

To do this, we find the derivatives of the functions $\mathrm{x}(\varphi), \mathrm{x}(\varphi), \mathrm{y}(\varphi), \mathrm{z}(\varphi)$ :

$x^{\prime}(\varphi)=R \sin \varphi ; y^{\prime}(\varphi)=R \cos \varphi ; z^{\prime}(\varphi)=2 b(\varphi-\pi)$.

We substitute the expression (7) in the formula (6):

$L_{C H}=\int_{\frac{3 \pi}{4}-\frac{\alpha}{2}}^{\pi} \sqrt{R^{2}+4 b^{2}(\varphi-\pi)^{2} d \varphi}$.

The choice of the spatial configuration of the SN section in the form (5) allows one to obtain the arc length in the form of an integral calculated in elementary functions. To do this, we replace the variables:

$\varphi=\pi-\xi ; d \varphi=-d \xi$ 
$\varphi_{1}=\frac{3 \pi}{4}-\frac{\alpha}{2} ; \varphi_{2}=\pi ;$

$\xi_{1}=\frac{\pi}{4}+\frac{\alpha}{2} ; \xi_{2}=0$.

In the formula (8) we swap the limits of integration, from the root we take out the constant $2 \mathrm{~b}$ outside the sign of the integral and, making replacements in accordance with (9), we get

$L_{C H}=2 b \int_{0}^{\frac{\pi}{4}+\frac{\alpha}{2}} \sqrt{\left(\frac{R}{2 b}\right)^{2}}+\xi^{2} d \xi$.

To simplify, we introduce the following notation: Then the integral (10) has the form

$\eta=\frac{R}{2 b} ; \beta=\frac{\pi}{4}+\frac{\alpha}{2}$.

Then the integral (10) has the form

$L_{C H}=2 b \int_{0}^{\beta} \sqrt{\eta^{2}+b \xi^{2}} d \xi$.

We calculate the integral (12) using integration by parts.

As a result, the length of the arc of the loop of the curve in section $\mathrm{CH}$ is

$L_{C H}=b\left[\beta \sqrt{\eta^{2}+\beta^{2}}+\eta^{2} \ln \left\{\frac{\sqrt{\eta^{2}+\beta^{2}+\beta}}{\eta}\right\}\right]$.

The entire loop of silk thread consists of eight sections of the type Sn. The length of the section AB is denoted by a (Fig. 1 a) Thus, the length of the entire loop will be determined by the formula

$L_{C H}=8 b\left[\beta \sqrt{\eta^{2}+\beta^{2}}+\eta^{2} \ln \left\{\frac{\sqrt{\eta^{2}+\beta^{2}+\beta}}{\eta}\right\}\right]+2 a$.

Where the parameters $\eta$ and $\beta$ are found by formulas (11), and the parameter $b$ is found by formula (4). We transform the formula (13):

$L_{C H}=8 b \beta \eta\left[\sqrt{1+\frac{\beta^{2}}{\eta^{2}}+\frac{\eta}{\beta}} \ln \left\{\sqrt{1+\frac{\beta^{2}}{\eta^{2}}}+\frac{\beta}{\eta}\right\}\right]+2 a$.

We denote $\psi=\frac{\beta}{\eta}=\frac{4 d}{R(\pi+2 \alpha)}$

Taking into account formulas (4) and (11), we obtain the formula for the length of a silk thread in a loop, taking into account the spatial configuration of this loop:

$L=R(\pi+2 \alpha)\left[\sqrt{1+\psi^{2}}+\frac{1}{\psi} \operatorname{Ln}\left(\sqrt{1+\psi^{2}}+\psi\right)\right]+2 \mathfrak{a}$.

\section{RESULTS AND DISCUSSION}

The adequacy of the developed model was assessed by comparing the calculated values and experimental measurements of the parameters of the knit fabric loop and was carried out by statistical methods using the Maple 9.5 command package. The results of calculations of the length of the thread in the loop and statistical characteristics (the coefficient of variation and the relative error of the average for natural silk threads are in the range of $1.7+2.8$ and $1.3+1.9 \%$, respectively) confirm the validity of the proposed mathematical model, taking into account spatial structure of this loop to determine its length. The deviation of the estimated length of the thread in the loop from the experimental for natural silk threads is in the range from 1.5 to $2.5 \%$. For comparison, natural silk thread than common textile yarn has a small percentage deviation $-1.0+1.2 \%$, since it was developed by an industrial method and the samples are connected from one species.

\section{CONClusion}

Thus, the proposed mathematical model for determining the length of the thread in the loop of a knitted fabric, worked out by alternating in each row of natural silk thread and textile yarn, allows at the stage of designing and developing the fabric structure to predict the necessary properties that 
determine the required quality without preliminary production of samples, and It has a sufficiently high accuracy in calculating the length of the thread in the loop for both the fabrics in question and for the fabrics of traditional textile yarns.

\section{REFERENCES}

[1] Tryevtsev A. V. Determination of the stiffness of the yarn in bending in order to find the loop parameter of the knitwear knitting by geometric parameters // Izv.vuzov. Textile industry technology. 1991. No.6. 71 - 77s.

[2] Guseva A.A. General technology of knitwear production. -Moscow: Legprombytizdat, 1987 .-- 296 p.

[3] Kudryavin L.A., Shalov I.I. Basics of knitwear technology. Moscow, 1998 - 496 s.

[4] Umarova M. N., Mukimov M. M. // Collection of materials of the Republican scientific and practical conference //. On receipt of knitwear from silk yarn. May 22-23, 2003. Namangan-58 -C.

[5] Umarova M. N., Isabaev A. E., Mukimov M. M. Influence of the linear density of the main thread on the physicomechanical properties of knitwear // Problems of the Textile Industry. - T., 2005. - No. 3. -54-56

Citation:UmarovaMavludahonNazirovna* (2020)" Modeling of Spatial Configuration of the Loop and Calculation of the Length of the Thread in the Loop of the Tunting Binding", International Journal of Research Studies in Electrical and Electronics Engineering(IJRSEEE), 6(3), pp.12-16. DOI: http://dx.doi. org/10.20431/2454-9436.0601003

Copyright:@ 2020 Authors, This is an open-access article distributed under the terms of the Creative Commons Attribution License, which permits unrestricted use, distribution, and reproduction in any medium, provided the original author and source are credited. 